\title{
VERTIGEM NO IDOSO: RELATO DE CASO
}

\section{Vertigo in elderly: a case report}

\author{
Marcelle Alpino Levandowski ${ }^{(1)}$, Valéria Kruskiewicz Bueno (2), \\ Luciana Lozza de Moraes Marchiori ${ }^{(3)}$, Juliana Jandre Melo ${ }^{(4)}$
}

\begin{abstract}
RESUMO
Tema: investigar a vertigem em paciente idosa com vários comprometimentos sistêmicos e avaliar a efetividade terapêutica para o tratamento da vertigem Posicional Paroxística Benigna (VPPB). Procedimentos: estudo de caso longitudinal de paciente do sexo feminino com vertigem submetido à terapia fonoaudiológica com exercícios de habituação vestibular. Resultados: no período de seis meses, observou-se melhora no quadro vertiginoso da paciente. Conclusão: a reabilitação vestibular com exercícios de habituação vestibular mostrou eficácia no tratamento da VPPB da paciente.
\end{abstract}

DESCRITORES: Vertigem; Qualidade de Vida; Equilíbrio

\section{INTRODUÇÃO}

Há uma disfunção do equilíbrio denominada tontura, sempre que existe um conflito na integração de informações sensoriais responsáveis pelo controle postural ${ }^{1}$. A tontura que, quando rotatória, é denominada vertigem, é uma sensação de perturbação do equilíbrio corporal, presente em uma infinidade de doenças, acometendo principalmente indivíduos idosos. Sua origem está correlacionada em $85 \%$ dos casos com distúrbios do sistema vestibular, ocorrendo a sua sintomatologia em geral, durante a movimentação da cabeça ou mudanças posturais $^{2,3}$.

A vertigem, conhecida como Vertigem Posicional Paroxística Benigna (VPPB) é uma das mais freqüentes alterações do sistema vestibular, e acomete principalmente a população idosa ${ }^{2,3}$. A característica clínica típica da VPPB é uma crise vertiginosa súbita, algumas vezes grave, de curta

(1) Fonoaudióloga do Centro Auditivo Telex de Londrina; Bolsista de Iniciação Científica na Universidade Norte do Paraná no ano de 2006; Especialista Psicopedagogia pelo Centro Universitário Filadélfia.

(2) Fonoaudióloga na Phono Clínica de Londrina; Voluntária de Iniciação Científica na Universidade Norte do Paraná no ano de 2006.

(3) Fonoaudióloga; Docente na Universidade Norte do Paraná; Doutora em Medicina e Ciências da Saúde pela Universidade Estadual de Londrina-Paraná.

(4) Fonoaudióloga; Docente na Universidade Norte do Paraná; Mestre em Fonoaudiologia pela Pontifícia Universidade Católica de São Paulo. duração, com desaparecimento completo do sintoma em menos de 45 segundos, tendo como movimentos tipicamente desencadeantes, os de deitar; levantar da cama, virar de lado quando deitado, movimentar a cabeça para olhar para cima ${ }^{4}$.

Geralmente, os pacientes conseguem identificar a posição que desencadeia a crise e passam a evitá-la constantemente, levando a alterações e/ou distúrbios posturais que pioram o quadro e aumentam a incapacidade funcional ${ }^{3,4}$. Por este motivo, são necessárias certas atitudes frente ao paciente vertiginoso. Dentre as intervenções realizadas em pacientes com VPPB estão os exercícios de habituação vestibular, os medicamentos sedativos labirínticos que atenuam a sintomatologia enquanto se aguarda uma regressão natural do quadro de VPPB; a ablação cirúrgica do canal semicircular posterior e as manobras de reposicionamento, tais como: a manobra liberadora de Semont e o reposicionamento canalicular de Epley ${ }^{5}$.

Vários estudos foram feitos para tentar verificar a eficácia do tratamento baseado em exercícios na vertigem. A partir de 1940, foram elaboradas formas de tratamento por meio de exercícios ${ }^{6,7}$, observando-se que vários pacientes ficavam curados com esses exercícios, não necessitando de outro tratamento associado ${ }^{8}$. A partir daí se passa a afirmar que os exercícios utilizados na reabilitação vestibular se baseavam mais no fenômeno de habituação, definido como um processo fisiológico caracterizado pelo declínio progressivo de respostas às estimulações repetitivas. 
Embora os exercícios de habituação vestibular sejam eficazes no tratamento da VPPB, salienta-se que para o sucesso deste tratamento é fundamental a cooperação do paciente. Os principais protocolos de reabilitação vestibular enfatizam a necessidade de personalização dos exercícios para cada paciente ${ }^{8,9}$. Com isso, percebe-se a necessidade de esclarecer a origem da vertigem para o tratamento fonoaudiológico de reabilitação vestibular ${ }^{10}$, principalmente na população idosa, observando a história do paciente, suas alterações sistêmicas e necessidades, visando melhorar sua qualidade de vida.

A partir destas colocações decidiu-se investigar a vertigem em paciente idosa com vários comprometimentos sistêmicos e avaliar a efetividade terapêutica para o tratamento da Vertigem Posicional Paroxística Benigna (VPPB), na mesma.

\section{APRESENTAÇÃO DO CASO}

Foi realizado um estudo longitudinal da paciente I.C.F.com 76 anos de idade, sexo feminino, que apresenta VPPB e outras doenças que podem associar-se a tontura. Apresentava queixa de plenitude auricular, tontura, sensação de flutuação, sensação de cabeça oca ou pesada, escurecimento de visão, fadiga, falta de concentração ou memória, vertigem postural, medo de andar sem o apoio das muletas.

Motivo de encaminhamento: encaminhada em 2006 pelo médico otorrinolaringologista para reabilitação vestibular; com confirmação de diagnóstico de síndrome vertiginosa.

Antecedentes pessoais com história de: dores cervicais decorrentes de espondilose, com pinçamento dos espaços inter-vertebrais entre C4-C5C6-C7; Hipertensão arterial; Hipertireoidismo; Diabetes mellitus; Colesterol; Osteoporose; Artrose e atrite; Cardiopatia, já tendo realizado cateterismo; Usuária de prótese no joelho; Usuária dos seguintes medicamentos: metformina, furosemida, metildopa; maleato de enalapril; dicloridrato de flunarizina e Hidroclorotiazida.

Com o objetivo de descrever o caso foram realizadas: Anamnese audiológica, audiometria e Imitanciometria, além de serem coletados dados do exame de vecto-eletronistagmografia e da radiografia da coluna cervical.

O projeto de pesquisa 0028/06 foi analisado e aprovado pela Comissão de ética da UNOPAR de acordo com a Resolução 196 de 10/10/1996 do Conselho Nacional de Saúde.

\section{RESULTADOS}

Resultados da vecto-eletronistagmografia: presença de nistagmo rotatório, acompanhado de vertigem com cabeça pendente à esquerda, sugestivo de ductolitíase do canal semicircular. As provas calóricas indicaram normorreflexia labiríntica bilateral; sem preponderância direcional e sem predomínio labiríntico; como também outros achados significantes.

Resultados da audiometria e imitanciometria: Os limiares auditivos apresentaram perda auditiva neurossensorial leve em $4000 \mathrm{~Hz}$ e $6000 \mathrm{~Hz}$ à esquerda e na freqüência de $4000 \mathrm{~Hz}$ à direita. Curva Timpanométrica do tipo " $A$ " bilateralmente com presença de reflexos estapedianos em ambas as orelhas e presença de recrutamento na freqüência de 4000 $\mathrm{Hz}$ bilateralmente.

\section{Tratamento:}

O tratamento fonoaudiológico realizado com a paciente a partir de maio de 2006 foi baseado em protocolos com exercícios de Cawthorne ${ }^{6}$, Cooksey ${ }^{7}$ e Herdman ${ }^{8}$, levando em consideração a grande dificuldade de mobilidade cervical da paciente no planejamento do tratamento.

$\mathrm{Na} 5^{\text {a }}$ sessão de terapia fonoaudiológica, a paciente referiu melhora no quadro vertiginoso passando inclusive a andar sem o apoio das muletas, apresentando diminuição na intensidade e na freqüência das crises vertiginosas no segundo mês de tratamento. Durante a reavaliação fonoaudiológica, seis meses após o inicio do tratamento, a paciente referiu a necessidade de dar continuidade à terapia fonoaudiológica para a manutenção de seu bem estar, uma vez que apresentou piora do quadro vertiginoso, após crise hipertensiva, ocorrida uma semana antes da citada reavaliação.

No mesmo período em que a paciente foi submetida ao tratamento fonoaudiológico, teve acompanhamento de profissionais dos cursos de Fisioterapia, Nutrição e Farmácia e dos médicos Otorrinolaringologista e Neurologista que fazem parte do projeto de reabilitação vestibular do curso de Fonoaudiologia da UNOPAR, além do acompanhamento do Cardiologista devido ao comprometimento cardiovascular.

\section{DISCUSSÃO}

A paciente idosa com VPPB apresentada neste estudo de caso é portadora de inúmeros comprometimentos sistêmicos que podem estar associadas à vertigem.

As doenças cervicais podem levar à insuficiência vértebro-basilar devido à compressão da artéria 
vertebral e diminuição do fluxo sanguíneo neste território, que pode provocar o aparecimento de sintomas labirínticos como vertigens ${ }^{8}$.

A hipertensão arterial e a insuficiência cardíaca podem causar alterações auditivas e vestibulares em decorrência do comprometimento periférico e/ ou central dos sistemas auditivo e/ou vestibular ${ }^{8-10}$. Nesse caso, a paciente apresentou piora da vertigem após crise hipertensiva, necessitando novamente da intervenção terapêutica para melhora do quadro clínico vertiginoso.

Disfunções do metabolismo, alterações dos hormônios ovarianos e alterações hormonais tireoideanas, além do uso de medicamentos para alterações metabólicas e circulatórias, relatados pela paciente também podem afetar a orelha interna e provocar tontura ${ }^{9-11}$.

Assim, pacientes que apresentam distúrbios associados (situação comum nos idosos) que potencialmente podem causar tontura, terão uma prevalência maior de vertigem - e um prejuízo ainda maior da qualidade de vida. Muitos destes distúrbios seriam passíveis de prevenção por meio do cultivo de hábitos saudáveis desde a juventude, o que adiaria ou retardaria da suas manifestações 10,11, conquistando-se assim o que se pode chamar de "compressão da morbidade", entendida como uma vida relativamente saudável e comprimindo as doenças em um curto período de tempo logo antes da morte ${ }^{11}$.

A revisão bibliográfica realizada para execução desse trabalho e os dados obtidos por intermédio do estudo de caso desta paciente servem para mostrar o intrincado complexo metabólico hormonal e circulatório e ate mesmo emocional, presentes na maioria da população idosa, trazendo complicações orgânicas, inclusive para a orelha interna, acarretando o aparecimento e o agravamento da vertigem ${ }^{12-14}$. Isto reforça a importância de um programa de reabilitação personalizada respeitando não apenas a localização da lesão, mas também as queixas do paciente, além de suas dificuldades e facilidades ${ }^{15}$.

Tendo-se em vista a expectativa de vida cada vez mais alta, vários estudos têm sido desenvolvidos de modo a contribuir para a melhoria da qualidade de vida na terceira idade, sendo consideradas as magnitudes e as diferenças de cada grupo sobre o que eles mesmos valorizam na busca de seu bem-estar ${ }^{16}$.

Para a manutenção do bem estar população idosa, por meio da minimização dos quadros vertiginosos que, muitas vezes, são variados devido às inúmeras alterações sistêmicas, é necessário se levar em consideração às necessidades individuais de cada paciente e suas limitações, além de se estar entrosado com a equipe.

A terapia de habituação vestibular contribuiu para a diminuição das crises vertiginosas e para a melhora da autoconfiança da paciente, em relação ao seu equilíbrio. Passou a andar sem o apoio das muletas, sendo que, a mesma relatou que embora tenha sentido, a princípio, dificuldade na realização dos exercícios propostos pela sensação vertiginosa que os mesmos desencadeavam, com o passar do tempo se sentiu mais segura durante o aparecimento das crises, que se tornaram menos freqüentes e com menor intensidade, minimizando inclusive o seu medo em relação a quedas.

\section{CONCLUSÃO}

Optou-se pela terapia fonoaudiológica com exercícios de habituação vestibular. Tais procedimentos proporcionaram à paciente redução no quadro vertiginoso, certamente melhorando sua qualidade de vida. Salienta-se, porém, que há necessidade de se estar constantemente observando a evolução clínica devido às inúmeras alterações sistêmicas que podem levar o paciente idoso a outros episódios vertiginosos.

\section{ABSTRACT}

Background: to investigate the vertigo in elderly woman with several systemic commitments and evaluate treatment effectiveness for Benign Paroxysmal Positional Vertigo (BPPV). Procedures: longitudinal clinical study of a female patient with vertigo submitted to speech and hearing therapy with vestibular habituation exercises. Results: after six months the patient had clinical improvement. Conclusion: vestibular rehabilitation with vestibular habituation exercises showed effectiveness in the treatment of this patient with BPPV.

KEYWORDS: Vertigo; Quality of Life; Equilibrium 


\section{REFERÊNCIAS}

1. Ganança FF, Castro ASO, Branco FC, Natour J. Interferência da tontura na qualidade de vida de pacientes com síndrome vestibular periférica. Rev Bras Otorrinolaringol. 2004; 70(1):97-101.

2. Ganança MM, Caovilla HH, Munhoz MSL, Silva MLG. Alterações da audição e do equilíbrio corporal no idoso. Rev Bras Med. 1999; 56(10):995-1011.

3. Teixeira LJ, Machado JNP. Manobras para o tratamento da vertigem posicional paroxística benigna: revisão sistemática da literatura. Rev Bras Otorrinolaringol. 2006; 72(1):130-9.

4. Resende CR, Taguchi CK, Almeida JG, Fujita RR. Reabilitação vestibular em pacientes idosos portadores de vertigem posicional paroxística benigna. Rev Bras Otorrinolaringol. 2003; 69(4):535-40.

5. Maia RA, Diniz FL, Carlesse A. Manobras de reposicionamento no tratamento da vertigem paroxística posicional benigna. Rev Bras Otorrinolaringol. 2001; (67)5:612-6.

6. Cawthorne T. The physiological basis for head exercises. J Chart Soc Psysiot. 1944; 30:106-7.

7. Cooksey FS. Rehabilitation in vestibular injuries. Proc R Soc Med. 1946; 39:273-5.

8. Herdman SJ. Vestibular rehabilitation. In: Baloh RW, Halmagy GM, organizador. Disorders of the vestibular system. Philadelphia: Davies; 1996. p. 583-97.
9. Segarra-Maegaki JAS, Taguchi CK. Estudo do benefício da reabilitação vestibular nas síndromes vestibulares periféricas. Pró-Fono. 2005; 17(1):3-11.

10. Tiensoli LO, Couto ER, Mitre El. Fatores associados à vertigem ou tontura em indivíduos com exame vestibular normal. Rev CEFAC. 2004; 6(1):94-100.

11. Marchiori LLM, Rego Filho EA, Matsuo T. Hipertensão como fator associado à perda auditiva. Rev Bras Otorrinolaringol. 2006; 72(4):533-40.

12. Marchiori LLM, Rego Filho EA. Queixa de vertigem e hipertensão arterial. Rev CEFAC. 2008; 9(1):116-21.

13. Gazzola JM, Aratani MC, Perracini M, Ganança MM, Ganança FF. Circunstâncias e conseqüências de quedas em idosos com vestibulopatia crônica. Rev Bras Otorrinolaringol. 2006; 72(3):388-93.

14. Ganança MM, Caovilla HH, Munhoz MSL, Silva MLG, Ganança FF, Ganança CF. Lidando com a vertigem posicional paroxística benigna. Acta AWHO. 2005; 23(2): 18-25.

15. Nishino LK, Ganança CF, Manso A, Campos $\mathrm{CAH}$, Korn GP. Reabilitação vestibular personalizada: levantamento de prontuários dos pacientes atendidos no ambulatório de otoneurologia da I.S.C.M.S.P. Rev Bras Otorrinolaringol. 2005; 71(4):440-7.

16. Vecchia R, Riuz T, Bocchi S, Corrente J. Qualidade de vida na terceira idade: um conceito subjetivo. Rev Bras Epidemiol. 2005; 8(3):246-53.

RECEBIDO EM: 05/12/2007

ACEITO EM: 08/07/2008

Endereço para correspondência:

Rua Belo Horizonte, 1399

Londrina - PR

CEP: 86020-060

Tel: (43) 3323-6068

E-mail: luciana.marchiori@.unopar.br 